\title{
Halo-Galaxy Lensing: A Full Sky Approach
}

\author{
Roland de Putter ${ }^{1}$, Masahiro Takada ${ }^{2}$ \\ ${ }^{1}$ Berkeley Lab \& University of California, Berkeley, CA 94720, USA \\ ${ }^{2}$ Institute for the Physics and Mathematics of the Universe (IPMU), \\ The University of Tokyo, Chiba 277-8582, Japan
}

(Dated: November 6, 2018)

\begin{abstract}
The halo-galaxy lensing correlation function or the average tangential shear profile over sampled halos is a very powerful means of measuring the halo masses, the mass profile, and the halo-mass correlation function of very large separations in the linear regime. We reformulate the halo-galaxy lensing correlation in harmonic space. We find that, counter-intuitively, errors in the conventionally used flat-sky approximation remain at a \% level even at very small angles. The errors increase at larger angles and for lensing halos at lower redshifts: the effect is at a few \% level at the baryonic acoustic oscillation scales for lensing halos of $z \sim 0.2$, and comparable with the effect of primordial non-Gaussianity with $f_{\mathrm{NL}} \sim 10$ at large separations. Our results allow to readily estimate/correct for the full-sky effect on a high-precision measurement of the average shear profile available from upcoming wide-area lensing surveys.
\end{abstract}

PACS numbers: $98.65,98.80$

\section{INTRODUCTION}

Dark matter halos hosting galaxies and galaxy clusters emerge as a result of gravitational amplification of tiny primordial fluctuations in the cold dark matter (CDM) dominated structure formation scenario. The weak lensing distortion effect on the shapes of background galaxies due to the halos is a powerful means of measuring the mass and the mass profile independent of the dynamical state and the uncertain relation between baryonic and dark matter distributions (e.g. see [1] for a thorough review). Weak lensing is now recognized as one of the most promising methods for constraining cosmology including the nature of dark energy (e.g. [2]), which is the primary motivation for both on-going and planned high precision weak lensing surveys, such as the CFHT Legacy Survey [35], the Hyper Suprime-Cam Weak Lensing Survey [3], the Dark Energy Survey (DES) [36], and ultimately the Large Synoptic Survey Telescope (LSST) [37], Euclid [4], and the Joint Dark Energy Mission (JDEM) [38].

There is a promising weak lensing technique, the so-called stacked lensing or halo-galaxy lensing correlation, which has been increasingly recognized as a statistically robust method since the first measurement a decade ago ([5, 6]; also see [7-12] for the recent measurements). The halo-galaxy lensing correlation can be measured first by measuring the tangential ellipticity/shear component of background galaxies with respect to the halo center in each halo region (galaxy or galaxy cluster selected beforehand) and then by averaging the tangential shear profiles over all the sampled halos that are tagged by a similar halo richness indicator and are in the same redshift range. Although lensing information for individual halos is lost, the halo-galaxy lensing correlation has several notable advantages. First, the average lensing profile becomes insensitive to substructures and asphericity of the individual halos and also to the projection effect, i.e. the contamination arising from uncorrelated large-scale structure along the line-of-sight. This is because these "contaminating signals" are averaged out via the stacking, under the assumption that the Universe is statistically homogeneous and isotropic. The stacked shear profile thus allows to extract the halo mass enclosed within a three-dimensional sphere of a given radius, which is an important quantity when using the halo mass function for constraining cosmology. Second, since the signal probed is linear in an estimator of lensing shear or galaxy ellipticity, the halo-galaxy lensing is more robust to systematic errors: the systematic errors, if relatively well behaved, are to some extend averaged out, in contrast to the cosmic shear measurements, where the measurement rests on the two-point correlation based methods and therefore the systematic errors may accumulate. For example, if a relatively clean selection of background galaxies is available, which is usually the case for cluster lensing [12], the stacked lensing is not contaminated by the intrinsic alignment of galaxy images, which is one of the potentially dominant systematic errors in the cosmic shear measurement.

Thus the halo-galaxy lensing allows a precise measurement of the mean halo mass and the mean mass profile, which is useful in calibrating the halo mass-observable relations [13]. The stacked shear profile can boost the signal-to-noise ratios at very small angular scales as well as very large scales, while only small solid angles or too small signals are available for individual halo lensing. The stacked lensing signals on small scales arise from the mass distribution gravitationally bound within each halo (the so-called one-halo term), while the large-scale signals arise from the surrounding mass distribution, such as filamentary structures, correlated with the sampled halos (the two-halo term) (see $[7,14]$ for the halo model based modeling). In particular, [15] recently studied that, for planned wide-area lensing surveys, the halo-mass correlation function at large angles can be in principle used to measure features of the baryonic 
acoustic oscillations and to probe the effect of primordial non-Gaussianity [16], which appear at projected radii greater than $100 \mathrm{Mpc}$ corresponding to 10 degree scales for lensing halos at $z \sim 0.2$.

In this paper we reformulate the halo-galaxy lensing correlation function in harmonic space, i.e. by employing the full-sky approach, since all the previous observational and theoretical works of halo-galaxy lensing have been done based on the flat-sky approximation (see $[17,18]$ for the study of the lensing power spectrum based on the full-sky approach). We study how the full-sky correction is relevant on angular scales probed by future wide-area surveys, and is compared with the BAO features and with the effect of primordial non-Gaussianity. We indeed find a non-trivial scale-dependent correction to the flat-sky approximation, and that counter-intuitively, the correction is not confined to large angles.

The structure of this paper is as follows. In Sec. II we reformulate the average tangential shear profile based on the full-sky approach. In Sec. III we compare the shear profiles computed from the full-sky and flat-sky approaches. Sec. IV is devoted to brief conclusions. Readers who want to skip the derivations could go straight to our main analytic results Eqs. (18) and (29) and then to Sec. III where we show our numerical results. Unless explicitly stated we assume cosmological parameters to match a flat, $\Lambda$-dominated CDM model that is consistent with the recent WMAP results [19].

\section{FORMULATION}

\section{A. Weak Lensing Shear on the Sky}

The bending of light by foreground mass, gravitational lensing, is described by a mapping of the position of a point on the sky with coordinates $\left(\tilde{x}^{1}, \tilde{x}^{2}\right)$ (in the absence of lensing) to a different position with coordinates $\left(x^{1}, x^{2}\right)$ (e.g. see [1] for a thorough review). We call $x^{i}$ the lens- or image-plane coordinates and $\tilde{x}^{i}$ the source-plane coordinates. The deflection or displacement vector on the sky is given by the gradient of a lensing potential $\psi$ :

$$
\tilde{x}^{i}=x^{i}-\psi^{; i},
$$

where $\psi$ is a line of sight projection of the gravitational potential and ";" denotes a contravariant derivative with respect to the image coordinates. For a finite-size source such as a galaxy, lensing causes its image to be distorted. The distortion is described by differences in deflection angles between different parts of a source galaxy image. The small displacement vector connecting the two points in the observed (or lens-plane) coordinates, $\delta x^{i}$, is mapped from the vector in the source plane, $\delta \tilde{x}^{i}$, as

$$
\delta \tilde{x}^{i}=\left(\delta^{i}{ }_{j}-\psi_{; j}^{; i}\right) \delta x^{j} .
$$

Although the above expressions are covariant and valid in any coordinate system, to define the shear components $\gamma_{1}$ and $\gamma_{2}$, it is convenient to work in a locally orthonormal system, i.e. $g_{i j}=\delta_{i j}$, where $\delta_{i j}$ is the Kronecker delta function. In such a coordinate system, where one does not have to distinguish between lower and upper indices, we define the shear by

$$
\delta \tilde{\mathbf{x}}=\left(\begin{array}{cc}
1-\kappa-\gamma_{1} & -\gamma_{2} \\
-\gamma_{2} & 1-\kappa+\gamma_{1}
\end{array}\right) \delta \mathbf{x}
$$

with

$$
\begin{aligned}
\kappa & \equiv \frac{1}{2} \nabla^{2} \psi, \\
\gamma_{1} & \equiv \frac{1}{2}\left(\psi_{; 11}-\psi_{; 22}\right), \\
\gamma_{2} & \equiv \psi_{; 12} .
\end{aligned}
$$

The convergence is a particularly informative quantity because it is a direct line of sight integral of the matter density fluctuation field $\delta_{m}$ (see appendix A).

The tangential shear around a halo is in the flat sky approach defined in a polar coordinate system with the halo at the origin. On the spherical sky, this needs to be generalized to a spherical coordinate system. Without loss of generality the halo center can be taken at the north pole due to the statistical isotropy of the Universe. In spherical coordinates, the metric is given by

$$
d s^{2}=d \theta^{2}+\sin ^{2} \theta d \phi^{2} .
$$


At each point on the sphere, this coordinate system defines a local basis $\left(\mathbf{e}_{\theta}, \mathbf{e}_{\phi}\right)$ which is orthogonal, but not orthonormal. Orthonormality can be realized by locally making the transformation to the coordinates $(\theta, \varphi)$, where $\varphi$ is defined by $d \varphi=\sin \theta d \phi$. We then define the tangential and transverse shears by in the $(\theta, \varphi)$ system setting

$$
\gamma_{T} \equiv-\gamma_{1}, \quad \gamma_{\times} \equiv \gamma_{2} \quad(\text { in }(\theta, \varphi) \text { system }) .
$$

It will be useful to have expressions for $\kappa, \gamma_{T}$ and $\gamma_{\times}$in terms of derivatives of the lensing potential with respect to $\theta$ and $\phi$. To derive these, we will first construct $\psi_{; i j}$ in the $(\theta, \phi)$ system and then use the fact that $\psi_{; i j}$ is a rank two tensor to transform to the $(\theta, \varphi)$ system. We thus start by calculating the covariant derivatives of $\psi$ with respect to $\theta$ and $\phi$. The first covariant derivative is a derivative of a scalar and therefore simply equals the partial derivative: $\psi_{; i}=\psi_{, i}$. The second derivative is the derivative of the covariant vector $\psi_{; i}$ and is given by

$$
\psi_{; i j}=\psi_{, i j}-\Gamma_{i j}^{k} \psi_{, k},
$$

where the Christoffel symbols are defined by

$$
\Gamma_{b c}^{a}=\frac{1}{2} g^{a d}\left(g_{d c, b}+g_{b d, c}-g_{b c, d}\right) .
$$

The non-vanishing Christoffel symbols are

$$
\begin{aligned}
& \Gamma_{\phi \phi}^{\theta}=-\sin \theta \cos \theta \\
& \Gamma_{\theta \phi}^{\phi}=\Gamma_{\phi \theta}^{\phi}=\frac{\cos \theta}{\sin \theta}
\end{aligned}
$$

and all others Christoffel symbols are zero. This gives

$$
\psi_{; i j}=\left(\begin{array}{cc}
\partial_{\theta}^{2} \psi & \sin \theta \partial_{\theta}\left(\frac{1}{\sin \theta} \partial_{\phi} \psi\right) \\
\sin \theta \partial_{\theta}\left(\frac{1}{\sin \theta} \partial_{\phi} \psi\right) & \partial_{\phi}^{2} \psi+\sin \theta \cos \theta \partial_{\theta} \psi
\end{array}\right), \quad(i, j=\theta, \phi) .
$$

We now transform to the orthonormal system, where the basis is transformed as $\mathbf{e}_{\phi} \cdot \mathbf{e}_{\phi} \neq 1 \rightarrow \mathbf{e}_{\varphi} \cdot \mathbf{e}_{\varphi}=1$, giving

$$
\psi_{; i j}=\left(\begin{array}{cc}
\partial_{\theta}^{2} \psi & \partial_{\theta}\left(\frac{1}{\sin \theta} \partial_{\phi} \psi\right) \\
\partial_{\theta}\left(\frac{1}{\sin \theta} \partial_{\phi} \psi\right) & \frac{1}{\sin ^{2} \theta} \partial_{\phi}^{2} \psi+\frac{\cos \theta}{\sin \theta} \partial_{\theta} \psi
\end{array}\right), \quad(i, j=\theta, \varphi) .
$$

Using Eqs. (4) and (6), we can now easily identify

$$
\begin{aligned}
\kappa & =\frac{1}{2}\left(\psi_{; \theta \theta}+\psi_{; \varphi \varphi}\right)=\frac{1}{2}\left(\partial_{\theta}^{2} \psi+\frac{1}{\sin ^{2} \theta} \partial_{\phi}^{2} \psi+\frac{\cos \theta}{\sin \theta} \partial_{\theta} \psi\right) \\
\gamma_{T} & =-\frac{1}{2}\left(\psi_{; \theta \theta}-\psi_{; \varphi \varphi}\right)=-\frac{1}{2}\left(\partial_{\theta}^{2} \psi-\frac{1}{\sin ^{2} \theta} \partial_{\phi}^{2} \psi-\frac{\cos \theta}{\sin \theta} \partial_{\theta} \psi\right) \\
\gamma_{\times} & =\psi_{; \theta \varphi}=\partial_{\theta}\left(\frac{1}{\sin \theta} \partial_{\phi} \psi\right) .
\end{aligned}
$$

Eqs. (12) will be the starting point of the derivations of the relation between tangential shear and projected mass and the relation between tangential shear and the angular power spectrum of the halo-mass cross correlation.

\section{B. Tangential Shear}

A quantity of great cosmological interest is the angle-averaged tangential shear $\left\langle\gamma_{T}\right\rangle(\theta)$, where in general the angle average of a quantity $\alpha$ is defined as

$$
\langle\alpha\rangle(\theta) \equiv \frac{1}{2 \pi} \int_{0}^{2 \pi} d \phi \alpha(\theta, \phi) .
$$

It follows from rotational symmetry that after stacking, there is no loss of information if we restrict ourselves to the angle-averaged shear and convergence $\left\langle\gamma_{T}\right\rangle(\theta),\left\langle\gamma_{\times}\right\rangle(\theta)$ and $\langle\kappa\rangle(\theta)$. 
A useful property of $\left\langle\gamma_{\times}\right\rangle(\theta)$ and $\left\langle\gamma_{T}\right\rangle(\theta)$ is that the former has to equal zero and the latter can conveniently be expressed in terms of the projected matter density. In the flat sky approximation [20-22],

$$
\left\langle\gamma_{T}\right\rangle(\theta)=\bar{\kappa}(<\theta)-\langle\kappa\rangle(\theta)
$$

where

$$
\bar{\kappa}(<\theta)=\frac{2}{\theta^{2}} \int_{0}^{\theta} d \theta^{\prime} \theta^{\prime}\langle\kappa\rangle\left(\theta^{\prime}\right)
$$

is the mean convergence within a circular disk with angular radius $\theta$. The convergence in turn is a direct line of sight integral of the matter overdensity (see appendix A).

We will now derive the full-sky equivalent of Eq. (14). Using Eqs. (12) and Eq. (13), we find

$$
\begin{array}{r}
\left\langle\gamma_{T}\right\rangle(\theta)=-\frac{1}{2}\left(\partial_{\theta}^{2}\langle\psi\rangle-\frac{\cos \theta}{\sin \theta} \partial_{\theta}\langle\psi\rangle\right) \\
\langle\kappa\rangle(\theta)=\frac{1}{2}\left(\partial_{\theta}^{2}\langle\psi\rangle+\frac{\cos \theta}{\sin \theta} \partial_{\theta}\langle\psi\rangle\right) .
\end{array}
$$

To relate the shear to the convergence, we need to define the mean convergence averaged within circular aperture of radius $\theta$ (the full-sky version of Eq. (15)),

$$
\begin{aligned}
\bar{\kappa}(<\theta) & \equiv \frac{1}{2 \pi(1-\cos \theta)} \int_{0}^{\theta} \sin \theta^{\prime} d \theta^{\prime} \int_{0}^{2 \pi} d \phi \kappa(\theta, \phi) \\
& =\frac{1}{1-\cos \theta} \int_{0}^{\theta} \sin \theta^{\prime} d \theta^{\prime}\langle\kappa\rangle\left(\theta^{\prime}\right) \\
& =\frac{\sin \theta}{2(1-\cos \theta)} \partial_{\theta}\langle\psi\rangle .
\end{aligned}
$$

Eqs. (16) and (17) above now allow us to write

$$
\left\langle\gamma_{T}\right\rangle(\theta)=2 \frac{\cos \theta}{1+\cos \theta} \bar{\kappa}(<\theta)-\langle\kappa\rangle(\theta) .
$$

This is the main result of this subsection. Note that in the small-angle limit, $\theta \ll 1$, the flat-sky result (14) is recovered.

\section{Average Tangential Shear Profile: Halo-Mass Correlation Function}

An even more useful observable is the so-called "stacked tangential shear" or "halo-galaxy correlation function", which is obtained by computing the tangential shear profile of background galaxy images relative to each halo [39], observationally represented by a galaxy or cluster, and then taking the average over all the sampled halos [5, 6, 23]. The stacked shear profile can be estimated as

$$
\left\langle\left\langle\gamma_{T}\right\rangle\right\rangle(\theta) \equiv \frac{1}{N_{h}} \sum_{\text {halos }}\left\langle\gamma_{T}\right\rangle(\theta),
$$

where $N_{h}$ is the total number of halos averaged over. The ensemble average of this quantity gives the halo-shear correlation function or the halo-mass correlation function:

$$
\left\langle\left\langle\gamma_{T}\right\rangle\right\rangle(\theta)=\left\langle\delta_{h}\left(\vec{\theta}^{\prime}\right) \gamma_{T}\left(\vec{\theta}^{\prime}+\vec{\theta}\right)\right\rangle=\left\langle\delta_{h}(0)\left\langle\gamma_{T}\right\rangle(\theta)\right\rangle,
$$

where $\delta_{h}(\vec{\theta})$ is the angular density fluctuation field of halos on the sky, and $\gamma_{T}$ is defined relative to the position $\left(\theta^{\prime}, \phi^{\prime}\right)$. The independence of $\theta^{\prime}$ and $\phi^{\prime}$ follows from rotational symmetry, which comes from the statistical isotropy of the universe.

By comparing the observed stacked shear to the value predicted by theory, one can learn about large scale structure and the background expansion history (e.g. see [7] for such a study). To calculate the theoretical $\left\langle\left\langle\gamma_{T}\right\rangle\right\rangle$, one first uses 
the halo-mass cross power spectrum $P^{h m}(k, z)$ and the background cosmology to construct the angular convergencehalo spectrum $C_{l}^{\kappa h}$ (see appendix A). Then, in the flat sky approximation, this angular spectrum is used to calculate $\left\langle\left\langle\gamma_{T}\right\rangle\right\rangle$ as a function of $\theta$ through

$$
\left\langle\left\langle\gamma_{T}\right\rangle\right\rangle(\theta)=\int \frac{l d l}{2 \pi} C_{l}^{h \kappa} J_{2}(l \theta)
$$

where $C_{l}^{h \kappa}$ is the angular power spectrum of cross-correlation between halos and the convergence field due to mass distribution surrounding the halos for a given source galaxy population, and $J_{n}$ is the $n$-th order Bessel function of the first kind. However, this relation is modified when the curvature of the sky is taken into account. Below we will derive the exact relation.

We start by expanding the halo density fluctuation field and the lensing potential field in spherical harmonics:

$$
\begin{aligned}
\delta_{h}(\theta, \phi) & =\sum_{l, m} a_{l m}^{h} Y_{l m}(\theta, \phi), \\
\psi(\theta, \phi) & =\sum_{l, m} a_{l m}^{\psi} Y_{l m}(\theta, \phi) .
\end{aligned}
$$

The spherical harmonics are

$$
Y_{l m}(\theta, \phi)=\sqrt{\frac{2 l+1}{4 \pi} \frac{(l-m) !}{(l+m) !}} P_{l}^{m}(\cos \theta) e^{i m \phi},
$$

with $P_{l}^{m}$ the associated Legendre polynomials. The angular power spectra are in general defined by

$$
\left\langle\left(a_{l m}^{X}\right)^{*} a_{l^{\prime} m^{\prime}}^{Y}\right\rangle=C_{l}^{X Y} \delta_{l l^{\prime}} \delta_{m m^{\prime}} .
$$

The tangential shear can according to Eqs. (12) and (22) be expanded as

$$
\gamma_{T}=-\frac{1}{2} \sum_{l m} a_{l m}^{\psi}\left(Y_{l m ; \theta \theta}(\theta, \phi)-Y_{l m ; \varphi \varphi}(\theta, \phi)\right)
$$

It is now straightforward to work out the stacked tangential shear:

$$
\begin{aligned}
\left\langle\left\langle\gamma_{T}\right\rangle\right\rangle(\theta) & =\left\langle\delta^{h}(0) \gamma_{T}(\theta, \phi)\right\rangle \\
& =-\frac{1}{2} \sum_{l m} Y_{l m}^{*}(0)\left(Y_{l m ; \theta \theta}(\theta, \phi)-Y_{l m ; \varphi \varphi}(\theta, \phi)\right) C_{l}^{\psi h} \\
& =-\frac{1}{2} \sum_{l} \sqrt{\frac{2 l+1}{4 \pi}}\left(Y_{l 0 ; \theta \theta}(\theta, \phi)-Y_{l 0 ; \varphi \varphi}(\theta, \phi)\right) C_{l}^{\psi h}
\end{aligned}
$$

(using $Y_{l m}(0)=\delta_{m, 0} \sqrt{(2 l+1) / 4 \pi}$ ). The second (covariant) derivatives of a scalar in the $(\theta, \varphi)$ coordinate system can be read off from Eq. (11) or (12), giving for the terms in parentheses

$$
\begin{aligned}
Y_{l 0 ; \theta \theta}(\theta, \phi)-Y_{l 0 ; \varphi \varphi}(\theta, \phi) & =\sqrt{\frac{2 l+1}{4 \pi}}\left(\partial_{\theta}^{2}-\frac{\cos \theta}{\sin \theta} \partial_{\theta}\right) P_{l}(\cos \theta) \\
& =\sqrt{\frac{2 l+1}{4 \pi}}\left(\sqrt{1-x^{2}} \frac{d}{d x}\left(\sqrt{1-x^{2}} \frac{d}{d x} P_{l}(x)\right)+x \frac{d}{d x} P_{l}(x)\right) \\
& =\sqrt{\frac{2 l+1}{4 \pi}}\left(1-x^{2}\right) \frac{d^{2}}{d x^{2}} P_{l}(x) \\
& =\sqrt{\frac{2 l+1}{4 \pi}} P_{l}^{2}(x), \quad x \equiv \cos \theta,
\end{aligned}
$$

where $P_{l}$ is the Legendre polynomial and $P_{l}^{2}$ is the associated Legendre polynomial with $m=2$. Since $\kappa=\frac{1}{2} \nabla^{2} \psi$ and $\nabla^{2} Y_{l m}=-l(l+1) Y_{l m}$,

$$
C_{l}^{\psi h}=-\frac{2}{l(l+1)} C_{l}^{\kappa h}
$$


Inserting Eq. (27) and Eqs. (28) into (26) gives the final result

$$
\left\langle\left\langle\gamma_{T}\right\rangle\right\rangle(\theta)=\sum_{l} C_{l}^{\kappa g} \frac{2 l+1}{4 \pi l(l+1)} P_{l}^{2}(\cos \theta)
$$

In the small-angle, large-l limit [17], $l \gg 1, \theta \ll 1$,

$$
P_{l}^{m}(\cos \theta) \approx(-1)^{m} \frac{(l+m) !}{(l-m) !} l^{-m} J_{m}(l \theta),
$$

so that for small angles, the flat-sky result (21) is recovered.

Eq. (29) is the main result of this paper as it gives the exact expression for the observed stacked shear profile in terms of the theoretical angular power spectrum of the mass-halo correlation.

\section{RESULTS: COMPARING FULL- AND FLAT-SKY APPROACHES}
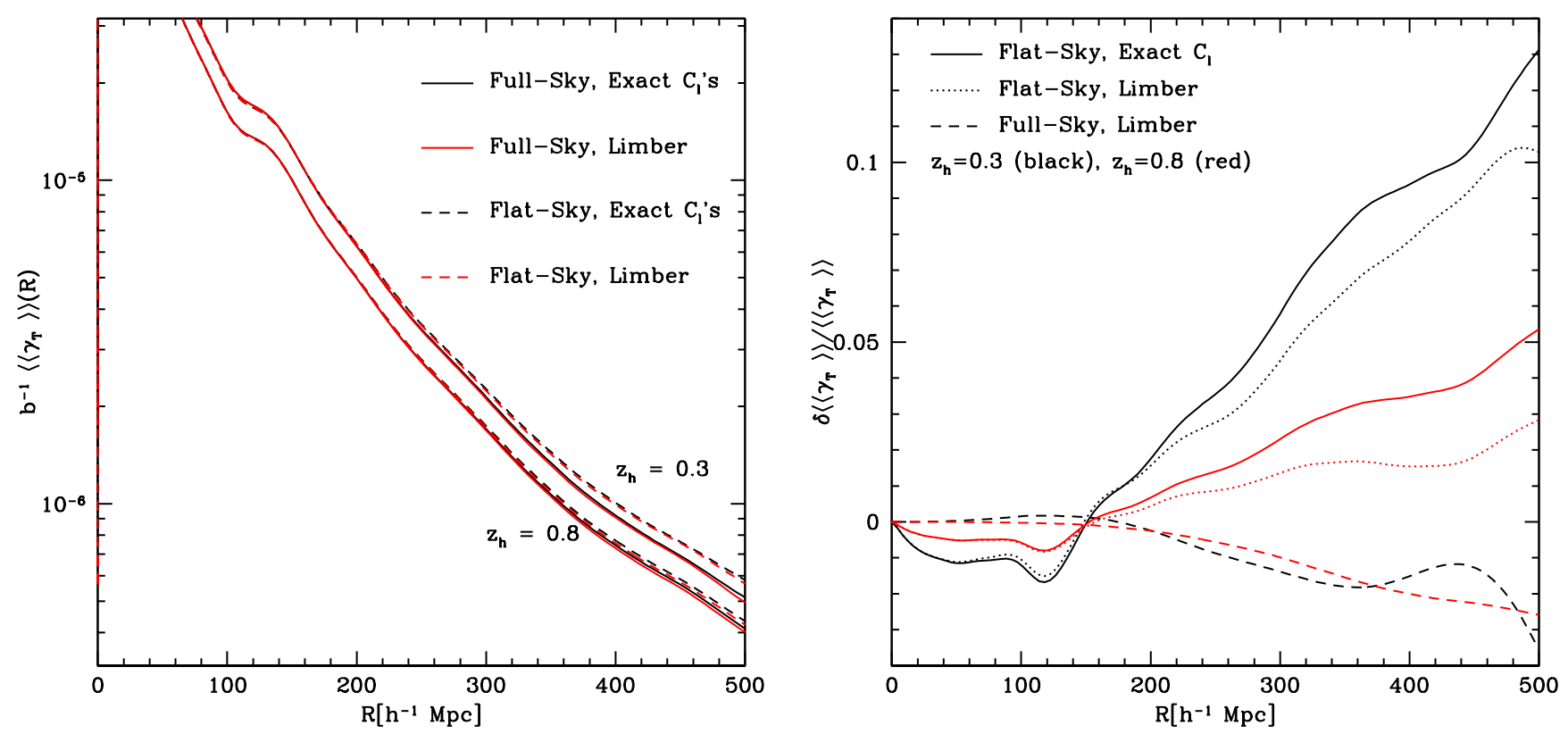

FIG. 1: Left panel: The average tangential shear profile (divided by bias) as a function of halo centric radii for lensing halos at redshifts $z_{h}=0.3$ and 0.8. The solid curves are the results computed from the full-sky approach, developed in this paper, while the dashed curves show the results from the flat-sky approximation. The curves denoted by "Limber" show the results using the Limber approximation for the calculation of the halo-mass power spectrum (see text for the details). Right panel: Relative differences between various approximations and the exact full-sky result. Note that the difference is defined as $\delta\left\langle\left\langle\gamma_{T}\right\rangle\right\rangle /\left\langle\left\langle\gamma_{T}\right\rangle\right\rangle \equiv\left\langle\left\langle\gamma_{T}\right\rangle\right\rangle_{\text {flat }} /\left\langle\left\langle\gamma_{T}\right\rangle\right\rangle_{\text {full }}-1$

Now that we have an exact, full-sky expression for the stacked shear profile, $\left\langle\left\langle\gamma_{T}\right\rangle\right\rangle(\theta)$ (see Eq. 29), we compare it to the result computed from the conventionally used flat-sky approximation. We do this by studying $\left\langle\left\langle\gamma_{T}\right\rangle\right\rangle$ for halos at two lensing redshifts $z_{h}=0.3$ and 0.8 . For the redshift distribution of source galaxies we assumed $z_{0} \approx 0.37$ for the functional form $n(z) \propto z^{2} \exp \left[-z / z_{0}\right]$ given by Eq. (7) in [24] such that the mean source redshift $\langle z\rangle=1$. Then we used the galaxies sitting in the redshift range $z=[1,1.5]$ as for source galaxies, yielding the mean redshift $\langle z\rangle \simeq 1.2$. For the matter power spectrum needed to compute the angular power spectrum $C_{l}^{h \kappa}$ we used the non-linear power spectrum, obtained by applying the HaloFit prescription [25] to the linear power spectrum, for a concordance $\Lambda \mathrm{CDM}$ model that is consistent with the WMAP 7-year results [19]. For the halo bias we simply assume a linear bias throughout this paper: the halo-mass correlation strengths are different from the mass correlation strengths by some constant factor. Although a more precise modeling of the halo-galaxy lensing requires to use a suite of N-body simulations, our approach is adequate enough, because the primary purpose of this paper is to study the impact of full-sky approach being compared with the conventionally used flat-sky results. 
In Fig. 1 we study the stacked shear profile as a function of comoving transverse distances from the halo center, $R \equiv \theta \chi_{h}$. Note that the comoving radial distances to halos at $z_{h}=0.3$ and 0.8 are $\chi_{h}=840$ and $1984 h^{-1} \mathrm{Mpc}$, respectively, for a flat $\Lambda \mathrm{CDM}$ model and therefore the comoving transverse length of $100 h^{-1} \mathrm{Mpc}$ corresponds to 6.82 and 2.89 degrees, respectively, for the two lensing redshifts. We also study the effect of the Limber approximation [26], which is often used in the literature to calculate the angular spectrum of halo-mass correlations $C_{l}^{h \kappa}$ (see Appendix $\mathrm{A}$ for the details).

The left panel of Fig. 1 compares the average tangential shear profile, computed from the full-sky approach, with those computed from various approximations, while the right panel shows the relative differences. The three approximations we consider are: flat-sky shear with exact $C_{l}^{h \kappa}$, flat-sky shear with Limber approximated $C_{l}^{h \kappa}$, and full-sky shear with Limber approximated $C_{l}^{h \kappa}$. The main interest of this paper is the comparison of the flat-sky approximation to the exact expression, so we focus now on the flat-sky with exact $C_{l}^{h \kappa}$ case. The first thing to notice is that the error caused by the flat-sky approximation is larger for $z_{h}=0.3$ than $z_{h}=0.8$, which can be explained by noting that a fixed transverse comoving distance to the line-of-sight corresponds to a larger angle for lower lensing redshifts. As for the scale-dependence of the difference, as expected, it starts at zero for $R=0$. Then, it starts to deviate linearly from zero even at small distances, with the flat-sky expression underestimating the signal. The perhaps counter-intuitive errors at small angles arise from the leading order correction in the asymptotic approximation relating the 2nd-order Legendre polynomial $P_{l}^{2}$ to the 2nd-order Bessel function in Eq. (30), as explained in detail in Appendix B. The difference reaches the $1 \%$ level around the BAO scale and then becomes greater at larger distances. The flat-sky expression overestimates the signal by more than $10 \%$ at $R \gtrsim 400 h^{-1} \mathrm{Mpc}$ for $z_{h}=0.3$. Thus we need to account for the full-sky effect for a \%-level high-precision measurement of the lensing-halo correlation, expected from upcoming wide-area lensing surveys.

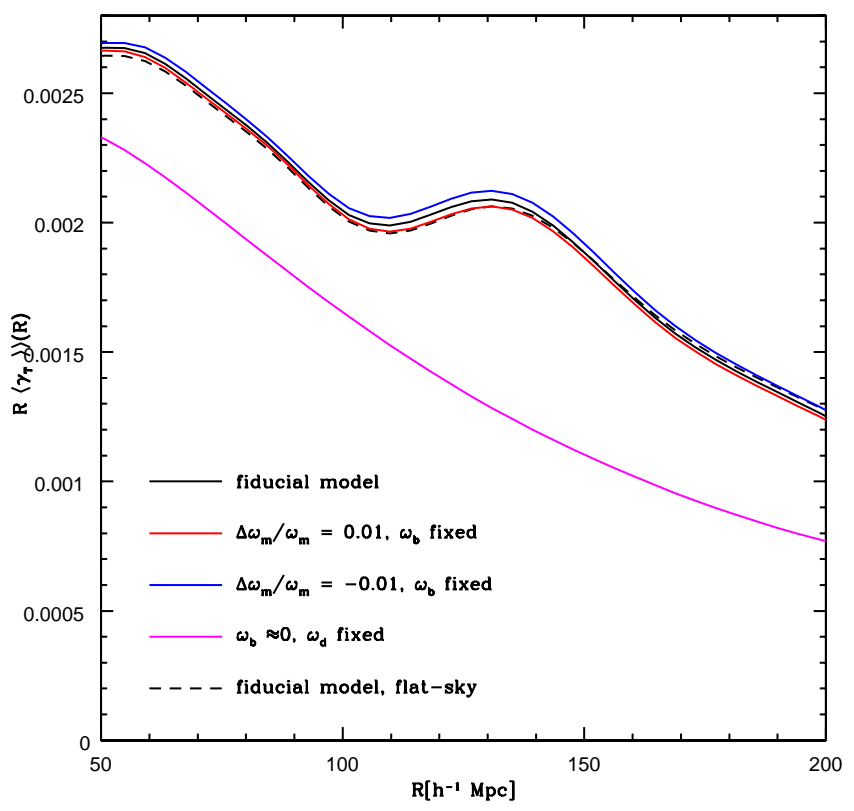

FIG. 2: The BAO feature in the stacked tangential shear profile (divided by bias) for $z_{h}=0.3$. We show the shear rescaled by a factor $R$ to make the feature come out more clearly. The red and blue lines correspond to a variation of $\pm 1 \%$ in the total matter density with the baryon density kept fixed. The magenta curve corresponds to $\omega_{b}=0$, giving no acoustic feature at all. The dashed line is for the fiducial model, but using the flat-sky approximation.

Recently [15] studied that the halo-galaxy lensing profile can be used to probe the BAO feature, appearing at $R \approx 147 \mathrm{Mpc}$, and to explore the effect of primordial non-Gaussianity, which become manifest at even larger scales. Since the error caused by the flat-sky approximation becomes significant at larger angles, it is useful to compare this error to these signals.

In Fig. 2, we show the stacked tangential shear profile (divided by bias) for $z_{h}=0.3$, zooming in on the BAO feature. The figure shows that the flat-sky error is comparable to a change in signal due to a variation of about $1 \%$ in the total matter density $\omega_{m}$ (i.e. $1 \%$ of the fiducial value for $\omega_{m}$ ), with the baryon density $\omega_{b}$ being fixed.

The local-type primordial non-Gaussianity parametrized by $f_{\mathrm{NL}}$ causes a scale-dependent bias in the halo-mass 

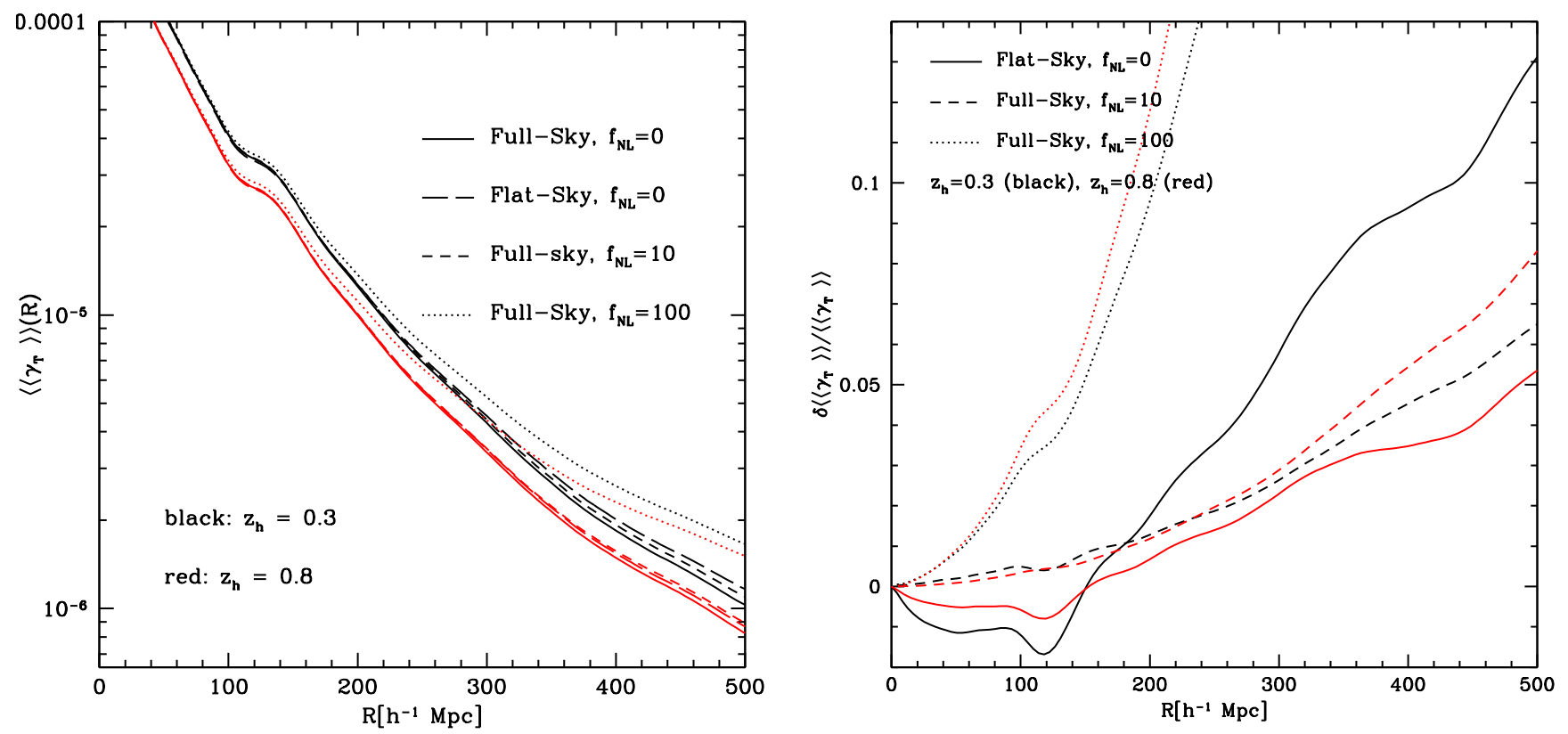

FIG. 3: The stacked tangential shear profile as a function of $f_{\mathrm{NL}}$ compared to the flat-sky result. We assume a linear bias $b_{1}=2$. Left panel: The profiles themselves. Right panel: The difference relative to the $f_{\mathrm{NL}}=0$, exact result. The error due to the flat-sky approximation is comparable to the effect of $f=\mathcal{O}(10)$.

correlation [16, 27-33]: the halo-mass cross power spectrum can be formally given as

$$
P^{h m}(k)=\left[b_{1}+\Delta b(k)\right] P^{m m}(k),
$$

where $P^{m m}(k)$ is the matter power spectrum. The scale-dependent bias is given by

$$
\Delta b(k) \equiv f_{\mathrm{NL}}\left(\frac{k_{\mathrm{NG}}(a)}{k}\right)^{2},
$$

where

$$
k_{\mathrm{NG}}(a)=\sqrt{\frac{3\left(b_{1}-1\right) \Omega_{m, 0} \delta_{c}}{a g(a)}} H_{0},
$$

which is roughly equal to the Hubble scale. In the above, $\delta_{c}=1.68$ is the critical overdensity for spherical collapse and $b_{1}$ is the bias in the absence of non-Gaussianity. The function $g(a)$ is the linear growth factor [34]: it is well approximated by the fitting formula

$$
g(a)=e^{\int \frac{d a}{a}\left(\left(\Omega_{m}(a)\right)^{\gamma}-1\right)},
$$

with $\gamma \approx 0.55$ (in GR, with a cosmological constant).

Since $C_{l}^{h \kappa}$ is a projection of $P^{h m}(k)$ (see Appendix A), it follows from Eq. (31) that $f_{\mathrm{NL}}$ affects the low $l$ part of the angular spectrum and therefore the stacked tangential shear at large separations. Assuming $b_{1}=2$, in Fig. 3 (left panel), we show the signal for $f_{\mathrm{NL}}=0, \pm 10, \pm 100$ and compare it to the flat-sky signal for $f_{\mathrm{NL}}=0$. In the right panel, we show the difference of non-zero $f_{\mathrm{NL}}$ and of the flat-sky expression for $f_{\mathrm{NL}}=0$, relative to the exact result for $f_{\mathrm{NL}}=0$. The error due to the flat-sky expression corresponds roughly to a change in $f_{\mathrm{NL}}$ of order 10 .

\section{CONCLUSIONS}

In this paper, we have developed a full-sky formalism for the halo-galaxy lensing correlation or the stacked lensing shear profile. We have updated the the flat-sky formula relating the tangential shear to convergence and found the 
exact expression for the stacked tangential shear $\left\langle\left\langle\gamma_{T}\right\rangle\right\rangle$ in terms of the angular halo-mass cross power spectrum. For the stacked tangential shear, we have confirmed by direct calculation that errors caused by the flat-sky approximation, which is currently used in the literature, are very small on scales relevant for current data ( $\lesssim 1 \%$ for comoving distance $R<20 h^{-1} \mathrm{Mpc}$ ). However, if in the future halo-galaxy lensing is used to constrain primordial non-Gaussianity by measuring the stacked tangential shear at separations of several hundreds of Mpc, the error due to the flat-sky approximation becomes relevant, it being of order $10 \%$ around $R=400 h^{-1} \mathrm{Mpc}$.

The size of the errors due to the flat-sky approximation, as shown in Figs. 1, 2 and 3, can be compared with Fig. 6 in [15], which shows that the fractional errors in measuring the halo-galaxy correlation function at these large scales for an all-sky lensing survey are at about 20-30\% level. Therefore the errors are well within the statistical measurement errors for existing and future lensing surveys. Nevertheless the exact, full-sky calculation is just as easy to do as the flat-sky approximation, our results can give a way to correct for or estimate the full-sky effect for future wide-area lensing surveys.

Acknowledgement: We thank A. Stebbins for useful discussion. This work is supported in part by World Premier International Research Center Initiative (WPI Initiative), MEXT, Japan. RdP has been supported in part by the Director, Office of Science, Office of High Energy Physics, of the U.S. Department of Energy under Contract No. DE-AC02-05CH11231.

Appendix A: angular spectra and Limber approximation
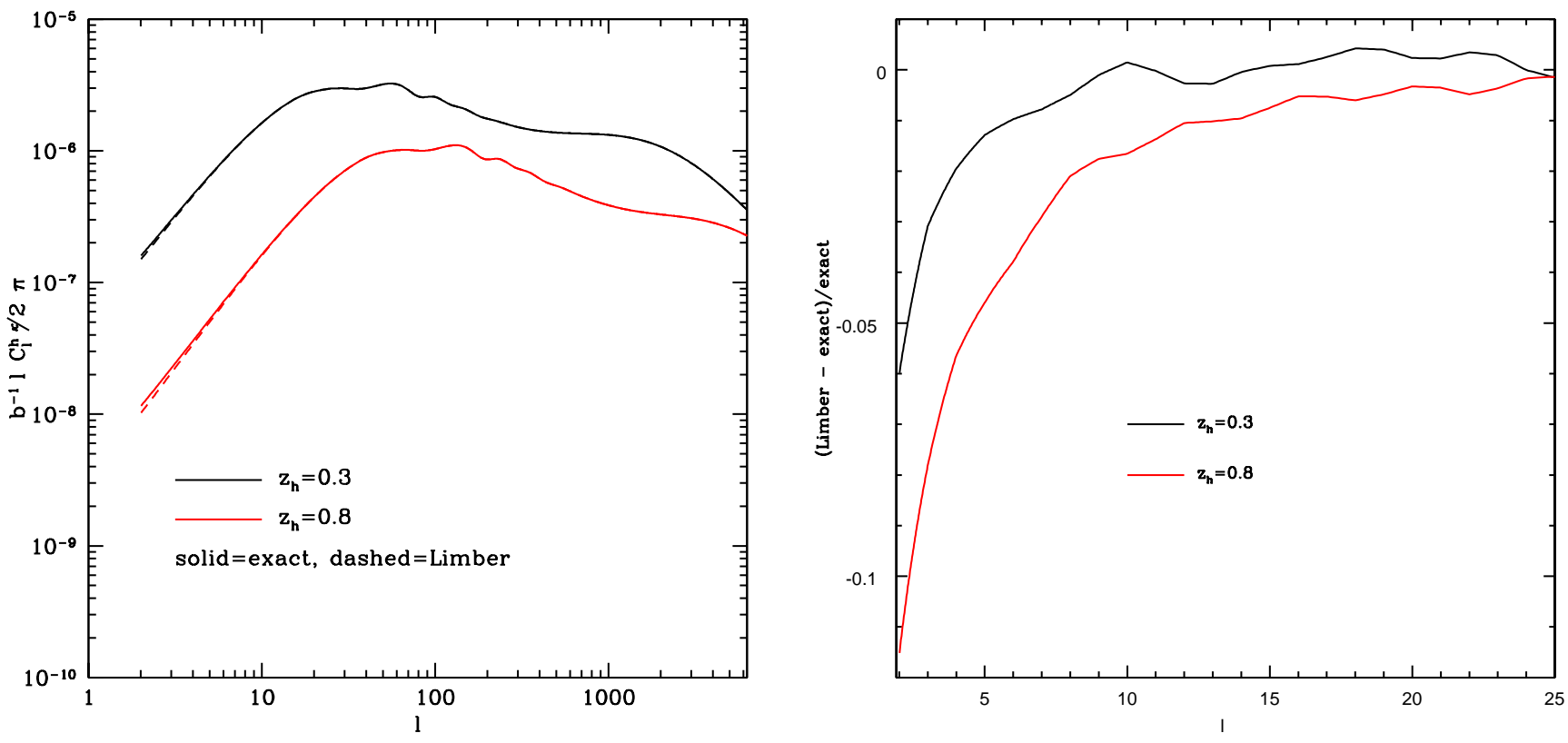

FIG. 4: Left panel: The full-sky result for the angular power spectrum of convergence and halo cross-correlation (divided by the linear halo bias) is compared with the result obtained from the Limber approximation for lensing redshifts $z_{h}=0.3$ and $z_{h}=0.8$ (see Eqs. [A6] and [A7]). Right: The relative differences.

To calculate $\left\langle\left\langle\gamma_{T}\right\rangle\right\rangle$, we need the angular cross spectrum of weak lensing convergence $\kappa$ with the (projected) halo density fluctuation field $\delta_{h}$. These quantities can both be expressed as line-of-sight projections of three dimensional quantities (the matter overdensity $\delta_{m}$ and the halo overdensity $\delta_{h}$ respectively):

$$
\kappa(\hat{n})=\int d \chi W^{\kappa}(\chi) \delta_{m}\left(\chi \hat{n}, \tau_{0}-\chi\right),
$$

and

$$
\delta_{h}(\hat{n})=\int d \chi W^{h}(\chi) \delta_{h}\left(\chi \hat{n}, \tau_{0}-\chi\right)
$$


with weight functions

$$
W^{\kappa}(\chi)=\frac{3}{2} H_{0}^{2} \Omega_{m, 0} \chi(1+z) \int_{z}^{\infty} d z_{s} \frac{\chi_{s}-\chi}{\chi_{s}}\left(\frac{n_{s}\left(z_{s}\right)}{n_{s, \text { tot }}}\right)
$$

and

$$
W^{h}(\chi)=H(z) \frac{n_{h}(z)}{n_{h, \text { tot }}},
$$

where $n_{s}\left(z_{s}\right)$ is the distribution of source galaxies, $\int d z_{s} n_{s}\left(z_{s}\right)=n_{s, \text { tot }}$, and $n_{h}(z)$ is the distribution of lens halos, $\int d z n_{h}(z)=n_{h, \text { tot }}$. Here $\chi$ is the comoving distance along the line of sight and we assume spatial flatness.

After expanding these fields in terms of spherical harmonics, the exact angular cross spectrum is given by

$$
C_{l}^{h \kappa}=\frac{1}{2 \pi^{2}} \int d \chi W^{h}(\chi) \int d \chi^{\prime} W^{\kappa}\left(\chi^{\prime}\right) \int d^{3} \vec{k} P^{h m}\left(k, \tau_{0}-\chi, \tau_{0}-\chi^{\prime}\right) j_{l}(k \chi) j_{l}\left(k \chi^{\prime}\right),
$$

where $P^{h m}\left(k, \tau, \tau^{\prime}\right) \delta\left(\vec{k}^{\prime}-\vec{k}\right) \equiv\left\langle\delta_{h}^{*}(\vec{k}, \tau) \delta_{m}\left(\vec{k}^{\prime}, \tau^{\prime}\right)\right\rangle$, and $j_{l}(x)$ denotes the $l$-th order spherical Bessel function.

When we can obtain a cross-correlation of the lensing convergence with halos at a single redshift $z_{h}$ (and comoving distance $\left.\chi_{h}\right)$, i.e. $n_{h}(z) / n_{h \text {,tot }}=\delta\left(z-z_{h}\right)$, the above expression reduces to

$$
C_{l}^{h \kappa}=\frac{1}{2 \pi^{2}} \int d^{3} \vec{k} j_{l}\left(k \chi_{h}\right) \int d \chi j_{l}(k \chi) W^{\kappa}(\chi) P^{h m}\left(k, \tau_{0}-\chi_{h}, \tau_{0}-\chi\right) .
$$

Expression (A6) is the one we use for our calculation of the exact halo-galaxy lensing correlation.

In the Limber approximation, the expression simplifies to

$$
\begin{aligned}
C_{l}^{h \kappa} & =H_{0}^{2} \frac{W^{\kappa}\left(\chi_{h}\right)}{\chi_{h}} P^{h m}\left(\frac{l+\frac{1}{2}}{\chi_{h}}, \tau_{0}-\chi_{h}\right) \\
& =\frac{3}{2} H_{0}^{2} \Omega_{m, 0}\left(1+z_{h}\right) \chi_{h}^{-1} P^{h m}\left(\frac{l+\frac{1}{2}}{\chi_{h}}, \tau_{0}-\chi_{h}\right)
\end{aligned}
$$

We show both the exact angular and the Limber approximated spectra (divided by bias) for $z_{h}=0.3$ and $z_{h}=0.8$ in the left panel of Fig. 4. In the right panel, we show the relative difference of the Limber spectra with the exact spectra. For low $l$, the Limber approximation underestimates the spectrum by up to $12 \%$ (for $z_{h}=0.3$ ). The difference becomes negligible at $l \sim 25$.

\section{Appendix B: Leading order correction for the flat-sky tangential shear profile}

The exact stacked tangential shear is given by

$$
\left\langle\left\langle\gamma_{T}\right\rangle\right\rangle(\theta)=\sum_{l} C_{l}^{\kappa g} \frac{2 l+1}{4 \pi l(l+1)} P_{l}^{2}(\cos \theta)
$$

and the flat-sky approximation gives

$$
\left\langle\left\langle\gamma_{T}\right\rangle\right\rangle(\theta)=\int \frac{l d l}{2 \pi} C_{l}^{\kappa g} J_{2}(l \theta) .
$$

The special functions involved are related by

$$
P_{l}^{2}(\cos \theta)=\frac{(l+2) !}{(l-2) !} l^{-2} J_{2}(l \theta)(1+\epsilon(l, \theta)),
$$

where $\epsilon$ is small and goes to zero as $\theta \rightarrow 0, l \rightarrow \infty$. For small $\theta$, the exact shear can thus be written

$$
\begin{aligned}
\left\langle\left\langle\gamma_{T}\right\rangle\right\rangle(\theta) & =\sum_{l} C_{l}^{\kappa g} \frac{2 l+1}{4 \pi l(l+1)} P_{l}^{2}(\cos \theta) \\
& =\left\{\sum \frac{1}{2 \pi} \frac{l+\frac{1}{2}}{l(l+1)} \frac{(l+2) !}{(l-2) !} l^{-2} J_{2}(l \theta) C_{l}\right\}(1+\mathcal{O}(\epsilon)) \\
& =\left\{\sum \frac{1}{2 \pi} l J_{2}(l \theta) C_{l}\left(1+\frac{3}{2} l^{-1}+\mathcal{O}\left(l^{-2}\right)\right)\right\}(1+\mathcal{O}(\epsilon)) \\
& \approx\left\{\int \frac{l d l}{2 \pi} J_{2}(l \theta) C_{l}\left(1+\frac{3}{2} l^{-1}+\mathcal{O}\left(l^{-2}\right)\right)\right\}(1+\mathcal{O}(\epsilon)) .
\end{aligned}
$$


Since the main contributions to the sum/integral come from $l \sim \theta^{-1}$, the correction to the flat-sky expression of order $l^{-1}$ inside the integral translates to a shear correction proportional to $\theta$. This effect alone would imply that the flat-sky approximation underestimates the shear at very low $\theta$. However, we also need to consider the $\mathcal{O}(\epsilon)$ term. We checked numerically that for fixed $l, \epsilon \propto \theta^{2}$ at least as long as $l \theta \ll 1$. However, the main contribution comes from $l \theta \sim 1$. We checked that for fixed $l \theta=x$ of order unity, $\epsilon=\epsilon(x / \theta, \theta) \propto \theta$ and $\epsilon<0$ so that this leads to an additional $\mathcal{O}(\theta)$ correction to the flat-sky shear. This effect on its own would imply that the flat-sky expression overestimates the true shear. We find numerically that the first effect wins (see Fig. 1) so that for low $\theta$, the relative difference between the flat-sky result and the exact result is negative and evolves linearly in $\theta$.

[1] M. Bartelmann and P. Schneider, Phys. Rep. 340, 291 (2001), arXiv:astro-ph/9912508.

[2] M. Takada and S. Bridle, New Journal of Physics 9, 446 (2007), arXiv:0705.0163.

[3] S. Miyazaki, Y. Komiyama, H. Nakaya, Y. Doi, H. Furusawa, P. Gillingham, Y. Kamata, K. Takeshi, and K. Nariai, in Society of Photo-Optical Instrumentation Engineers (SPIE) Conference Series (2006), vol. 6269 of Society of Photo-Optical Instrumentation Engineers (SPIE) Conference Series.

[4] A. Refregier, A. Amara, T. D. Kitching, A. Rassat, R. Scaramella, J. Weller, and f. t. Euclid Imaging Consortium, arXiv:1001.0061.

[5] P. Fischer, T. A. McKay, E. Sheldon, A. Connolly, A. Stebbins, J. A. Frieman, B. Jain, M. Joffre, D. Johnston, G. Bernstein, et al., Astron. J. 120, 1198 (2000), arXiv:astro-ph/9912119.

[6] T. A. McKay, E. S. Sheldon, J. Racusin, P. Fischer, U. Seljak, A. Stebbins, D. Johnston, J. A. Frieman, N. Bahcall, J. Brinkmann, et al., ArXiv Astrophysics e-prints (2001), arXiv:astro-ph/0108013.

[7] J. Guzik and U. Seljak, Mon. Not. R. Astron. Soc. 321, 439 (2001), arXiv:astro-ph/0007067.

[8] C. M. Hirata, R. Mandelbaum, U. Seljak, J. Guzik, N. Padmanabhan, C. Blake, J. Brinkmann, T. Budávari, A. Connolly, I. Csabai, et al., Mon. Not. R. Astron. Soc. 353, 529 (2004), arXiv:astro-ph/0403255.

[9] R. Mandelbaum, A. Tasitsiomi, U. Seljak, A. V. Kravtsov, and R. H. Wechsler, Mon. Not. R. Astron. Soc. 362, 1451 (2005), arXiv:astro-ph/0410711.

[10] R. Mandelbaum, U. Seljak, G. Kauffmann, C. M. Hirata, and J. Brinkmann, Mon. Not. R. Astron. Soc. 368, 715 (2006), arXiv:astro-ph/0511164.

[11] E. S. Sheldon, D. E. Johnston, R. Scranton, B. P. Koester, T. A. McKay, H. Oyaizu, C. Cunha, M. Lima, H. Lin, J. A. Frieman, et al., Astrophys. J. 703, 2217 (2009), arXiv:0709.1153.

[12] N. Okabe, M. Takada, K. Umetsu, T. Futamase, and G. P. Smith, Publ. Astron. Soc. Japan 62, 811 (2010), arXiv:0903.1103.

[13] E. Rozo, R. H. Wechsler, E. S. Rykoff, J. T. Annis, M. R. Becker, A. E. Evrard, J. A. Frieman, S. M. Hansen, J. Hao, D. E. Johnston, et al., Astrophys. J. 708, 645 (2010), arXiv:0902.3702.

[14] D. E. Johnston, E. S. Sheldon, R. H. Wechsler, E. Rozo, B. P. Koester, J. A. Frieman, T. A. McKay, A. E. Evrard, M. R. Becker, and J. Annis, arXiv:0709.1159.

[15] D. Jeong, E. Komatsu, and B. Jain, Phys. Rev. D 80, 123527 (2009), arXiv:0910.1361.

[16] N. Dalal, O. Doré, D. Huterer, and A. Shirokov, Phys. Rev. D 77, 123514 (2008), arXiv:0710.4560.

[17] A. Stebbins, arXiv:astro-ph/9609149.

[18] W. Hu, Phys. Rev. D 62, 043007 (2000), arXiv:astro-ph/0001303.

[19] E. Komatsu, K. M. Smith, J. Dunkley, C. L. Bennett, B. Gold, G. Hinshaw, N. Jarosik, D. Larson, M. R. Nolta, L. Page, et al., arXiv:1001.4538.

[20] N. Kaiser and G. Squires, Astrophys. J. 404, 441 (1993).

[21] G. Squires and N. Kaiser, Astrophys. J. 473, 65 (1996), arXiv:astro-ph/9512094.

[22] J. Miralda-Escude, in Astrophysical Applications of Gravitational Lensing, edited by C. S. Kochanek \& J. N. Hewitt (1996), vol. 173 of IAU Symposium, p. 131.

[23] E. S. Sheldon, J. Annis, H. Böhringer, P. Fischer, J. A. Frieman, M. Joffre, D. Johnston, T. A. McKay, C. Miller, R. C. Nichol, et al., Astrophys. J. 554, 881 (2001), arXiv:astro-ph/0103029.

[24] M. Takada and B. Jain, MNRAS (2004), arXiv:astro-ph/0310125.

[25] R. E. Smith, J. A. Peacock, A. Jenkins, S. D. M. White, C. S. Frenk, F. R. Pearce, P. A. Thomas, G. Efstathiou, and H. M. P. Couchman, Mon. Not. R. Astron. Soc. 341, 1311 (2003), arXiv:astro-ph/0207664.

[26] D. N. Limber, Astrophys. J. 119, 655 (1954).

[27] S. Matarrese and L. Verde, Astrophys. J. Lett. 677, L77 (2008), arXiv:0801.4826.

[28] A. Slosar, C. Hirata, U. Seljak, S. Ho, and N. Padmanabhan, Journal of Cosmology and Astro-Particle Physics 8, 31 (2008), arXiv:0805.3580.

[29] N. Afshordi and A. J. Tolley, Phys. Rev. D 78, 123507 (2008), arXiv:0806.1046.

[30] A. Taruya, K. Koyama, and T. Matsubara, Phys. Rev. D 78, 123534 (2008), arXiv:0808.4085.

[31] V. Desjacques, U. Seljak, and I. T. Iliev, Mon. Not. R. Astron. Soc. 396, 85 (2009), arXiv:0811.2748.

[32] A. Pillepich, C. Porciani, and O. Hahn, Mon. Not. R. Astron. Soc. 402, 191 (2010), arXiv:0811.4176.

[33] M. Grossi, L. Verde, C. Carbone, K. Dolag, E. Branchini, F. Iannuzzi, S. Matarrese, and L. Moscardini, Mon. Not. R. Astron. Soc. 398, 321 (2009), arXiv:0902.2013.

[34] E. V. Linder, Phys. Rev. D 72, 043529 (2005), arXiv:astro-ph/0507263. 
[35] http://www.cfht.hawaii.edu/Science/CFHLS/

[36] http://www.darkenergysurvey.org

[37] http://www.lsst.org

[38] http://jdem.gsfc.nasa.gov/

[39] Here we mean by "relative to" that we calculate the tangential shear in a coordinate system that has the halo at the north pole. 